\title{
Re-assessment of the Accretion Disc Clock in Hercules X-1
}

\author{
E. Jurua ${ }^{1,2}$, M. Still ${ }^{3}$, P. J. Meintjes ${ }^{1}$, P. A. Charles ${ }^{4}$ and \\ S. K Anguma ${ }^{1}$ \\ ${ }^{1}$ University of the Free State, P.O.Box 334, Bloemfontein 9300, South Africa \\ ${ }^{2}$ Mbarara University of Science and Technology, P.O.Box 1410, Mbarara, Uganda \\ ${ }^{3}$ NASA Ames Research Center M/S 244-30, Moffett Field, CA 94035, USA \\ ${ }^{4}$ South African Astronomical Observatory, P.O. Box 9, Observatory 7935, South Africa, \\ University of Cape Town, Private Bag X3, Rondebosch 7701, South Africa, and School of \\ Physics \& Astronomy, University of Southampton, Southampton SO17 1BJ, JK
}

\begin{abstract}
The Galactic neutron star X-ray binary Her X-1 shows a 35-day photometric modulation. Detected across a broad energy range, the modulation is prevalent in X-rays, cycling between low and high states. Since the discovery of the 35-day cycle of Her X-1, the X-ray source has entered and returned from an extended anomalous low state on at least seven occasions. Employing Rossi X-ray Timing Explorer All-Sky Monitor observations, prior predictions that Her X-1 would return from the 2003 - 2004 anomalous low state with a new precession period and accretion luminosity are verified. However, the precession has not been as coherent over recent years as predicted. Potentially there have been more minor anomalous low states between 2004 and the present day.
\end{abstract}

Keywords. accretion:accretion discs - binaries:eclipsing - star:individual (Hercules X-1) stars:neutron

\section{Introduction}

Hercules X-1 (Her X-1) is an eclipsing X-ray binary containing a $1.4 M_{\odot}$ (Reynolds et al. 1997) pulsar in orbit about a $2.2 M_{\odot}$ A7 Roche-lobe filling star (Middleditch \& Nelson 1976; Leahy \& Scott 1998), known optically as the variable HZ Her. Her X-1 has a pulse period of $1.24 \mathrm{~s}$ and an orbital period of 1.7 days (Tananbaum et al. 1972). In addition, it displays a $\sim 35$-day superorbital period detected over a variety of wavelengths (Giacconi et al. 1973, Leahy \& Marshall 1999, Scott, Leahy \& Wilson 2000). Optical photometric variations show a 1.62-day beat period between the superorbital and the orbital periods of Her X-1.

Since the discovery of the 35-day cycle of Her X-1, there have been occasions when it failed to reach its high state, i.e. it missed a number of consecutive 35-day cycles, a state called the Anomalous Low State (hereafter ALS). Still \& Boyd 2004 (hereafter SB04) showed that after each ALS, Her X-1 turns on with a different precession period and main-on state count rate; accompanied by a change in precession period and the subsequent period change is correlated with the accretion flux. SB04 predicted that Her X-1 would return from the 2003 - 2004 ALS with a new precession period and epoch averaged peak main-on flux. The purpose of this paper is to test these predictions.

\section{Observations}

The RXTE ASM consists of three scanning shadow cameras which give regular coverage of the whole sky (Levine et al., 1996). Each camera is sensitive to X-rays in the range 
1.5 - $12 \mathrm{keV}$, which is sub-divided into three energy bands corresponding to $\mathrm{A}=1.5$ $3 \mathrm{keV}, \mathrm{B}=3-5 \mathrm{keV}$ and $\mathrm{C}=5-12 \mathrm{keV}$. The ASM data consists of a number of dwells taken for each source per day with an exposure time of about 90 seconds once every 90 minutes. In this paper, RXTE ASM light-curves of Her X-1 are used to determine how the precession period has evolved since the 2003 - 2004 ALS.

\section{Results}
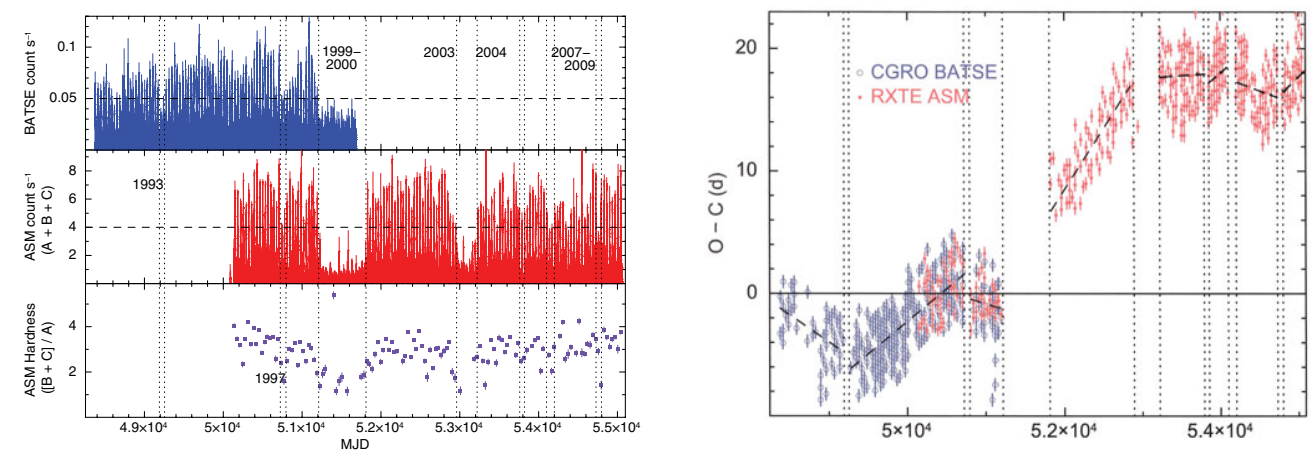

Figure 1. Left: Light curve of RXTE ASM 24 hour average count rates from Her X-1 for the period 1996 to 2009 shown in the middle panel. In the top panel is the CGRO BATSE one day average count rates between 1991 and 1999 (SB04) and the bottom panel is the ASM hardness ratio calculated from the daily dwells. In this Figure, the dotted vertical lines represent the start and stop times of each ALS, while the dashed horizontal lines represent the count rate threshold used to analyse the $\mathrm{O}$ - C. Right: A plot of $\mathrm{O}-\mathrm{C}$ time series of the main-on states in the Her $\mathrm{X}-1$ precession cycle. The linear least-square fits to the $\mathrm{O}-\mathrm{C}$ diagram are represented by the dashed lines for each epoch. Additional data obtained from SB04

A plot of the lightcurve of Her X-1 is shown in Figure 1. In this figure each of the spikes corresponds to the main-on phase of the 35 day cycle. ALS epochs, where the 35 day cycle is suppressed, are bounded by the vertical dotted lines. From this lightcurve we also note that Her X-1 entered three minor ALS after the 2003 - 2004 ALS and is currently in the eighth epoch of its normal high state (see Table 1 for the periods of the epochs of normal high state). Using the three ASM energy bands, the hardness ratio was calculated as 35-day averages and is plotted in the third panel of Figure 1 as a function of time, i.e. Modified Julian Date (MJD). To improve on the error margins, daily-dwell count rates with errors $\geqslant 1$ were filtered as well as hardness ratios with errors $\geqslant 1$. There is a possible relation between the hardness ratio and the ASM count rates with the hardness ratio softening during the low states.

Leahy and Igna (2010) applied a cross-corrrelation method to deternime the precession cycle lengths of Her X-1. In this paper, the average precession period during Epochs 5, 6, 7 and 8, defined in Table 1, were determined using an approach similar to that followed by SB04. The main-on turn-on times, historically used to measure the disc precession period, are not periodic and therefore we employ instead a measure of the mid-point of each main-on state, $T_{\text {mid }}$. Over the first years of the RXTE mission, these times can be characterised by a linear ephemeris, i.e. the reference ephemeris adopted (SB04) is

$$
T_{\mathrm{mid}}=\text { MJD } 50986.7+34.79 E_{35},
$$

where $E_{35}$ is an integer, i.e. the precession cycle number. Using an arbitrary threshold of 3 counts $\mathrm{s}^{-1}$ for the fifth, sixth, seventh and eighth epochs of normal high state, each 
data point greater than 3 counts $\mathrm{s}^{-1}$ was used to calculate and plot the "observed minus calculated" (O - C) curve of the precession cycle (Fig 2). In this approach, the quantity subtracted from the ASM times to produce the $\mathrm{O}-\mathrm{C}$ time series is the nearest $T_{\text {mid }}$ to each daily dwell. Individual main-ons are represented by many data points in the $\mathrm{O}-\mathrm{C}$ curve and the period is calculated from a linear least-squares fit to the $\mathrm{O}-\mathrm{C}$ diagram. The precession periods obtained for these epochs are given in Table 1. The derivative, $\dot{P}_{35}$, is obtained from a quadratic fit to the O - C diagram and is also given in Table 1.

The values of $\dot{P}_{35}$ are consistent with zero, within the $1-\sigma$ error, and so we use the linear fit for the ephemeris. Therefore the precession ephemeris for the turn-on $T_{\text {on }}$ for the fifth, sixth, seventh and eighth epochs are respectively given by

$T_{\text {on }}=$ MJD $53160(2)+34.85 E_{35} ; \quad T_{\text {on }}=$ MJD $53851(2)+35.28 E_{35} ;$

$T_{\text {on }}=$ MJD $54203(2)+34.65 E_{35} ; \quad T_{\text {on }}=$ MJD $54758(2)+34.99 E_{35}$

where a systematic uncertainty of two days is allowed in each case.

Folding the photometric data from each epoch over the precession period and averaging it into 50 phase bins at intervals of 0.02 , the count rates were plotted as a function of the precession phase, $\phi_{35}$ (Figure 3). Relative to the ephemerides presented in SB04 and section 3 , the main-on turn-on occurs at $\phi_{35}=0.0$. SB04 determined the brightest flux attained during the 35-day cycle by measuring the peak of a Gaussian fit of the folded light curve between $\phi_{35}=0.0$ and $\phi_{35}=0.4$. But the folded lightcurves shown in Figure 3 reveal that the 35 -day cycles are not symmetric between $\phi_{35}=0.0$ and $\phi_{35}=0.4$. It is therefore appropriate to integrate the folded light curve over time (35-day cycle), which defines the fluence. In this case the lightcurve between $\phi_{35}=0.0$ and $\phi_{35}=0.4$, i.e. the main-on, was considered for all the epochs and the values obtained for the integrated flux $F_{\text {int }}$ are shown in Table 1 . We verify that the positive correlation between the precession period and flux $F_{\text {int }}$ suggested by SB04 has continued to the present day.
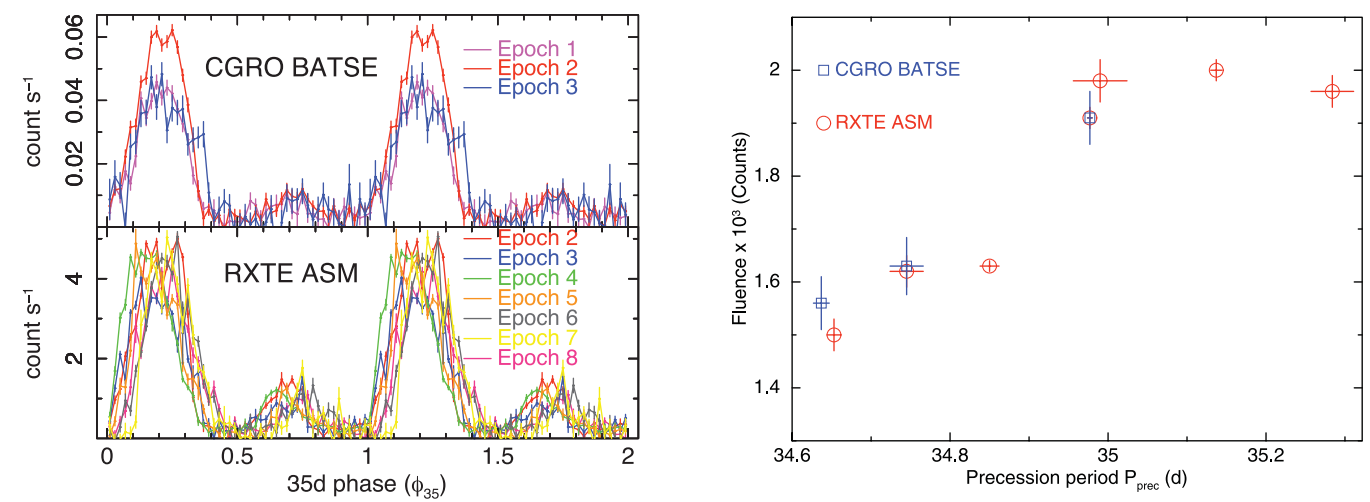

Figure 2. Left: The 24 hour averages folded over the 35-day cycle using the appropriate 35-day ephemeris. Additional data obtained from SB04. Right: The Fluence between $\phi_{35}=0.0$ and $\phi_{35}=0.4$ as a function of precession period. The CGRO BATSE flux has been scaled to fit the RXTE flux. Additional data obtained from SB04.

\section{Discussion}

SB04 determined the precession periods of Her X-1 for the first four epochs prior to 2004 and found that each ALS was accompanied by an unresolved change in precession period. New precession periods and fluxes have been obtained for epochs 5, 6, 7 and 8 (see Table 1), in agreement with the predictions of SB04. This is also in agreement with Leahy and Igna (2010) who shows that the precession cycle length vary from 33.2 
Table 1. Precession period $P_{35}$, derivative $\dot{P}_{35}$, and Fluence for five epochs of Her X-1 precession cycle.

\begin{tabular}{|c|c|c|c|c|c|c|c|}
\hline \multirow[b]{2}{*}{ Epoch } & \multicolumn{2}{|c|}{ CYCLE } & \multicolumn{2}{|c|}{ MJD } & \multirow{2}{*}{$\begin{array}{c}P_{35} \\
\text { (days) }\end{array}$} & \multirow{2}{*}{$\begin{array}{c}\dot{P}_{35} \\
\left(\text { days day }^{-1}\right)\end{array}$} & \multirow{2}{*}{$\begin{array}{l}\text { Fluence }\left(F_{\text {int }}\right) \\
\times 10^{3} \text { Counts }\end{array}$} \\
\hline & Start & Stop & Start & Stop & & & \\
\hline $1 \ldots$ & -75 & -52 & 48,377 & 49,213 & $34.637 \pm 0.010$ & $-(3 \pm 1) \times 10^{-7}$ & $0.018 \pm 0.001$ \\
\hline $2 \ldots$ & -49 & -8 & 49,282 & 50,708 & $34.977 \pm 0.003$ & $(3 \pm 2) \times 10^{-8}$ & $0.022 \pm 0.001^{a}, 1.91 \pm 0.04^{b}$ \\
\hline $3 \ldots$ & -5 & +6 & 50,813 & 51,195 & $34.745 \pm 0.021$ & $-(7 \pm 7) \times 10^{-10}$ & $0.019 \pm 0.002^{a}, 1.62 \pm 0.05^{b}$ \\
\hline $4 \ldots$ & +26 & +54 & 51,892 & 52,866 & $35.137 \pm 0.007$ & $1 \times 10^{-7}$ & $2.00 \pm 0.04$ \\
\hline $5 \ldots$ & +64 & +80 & 53,160 & 53792 & $34.850 \pm 0.012$ & $(6 \pm 4) \times 10^{-9}$ & $1.63 \pm 0.04$ \\
\hline $6 \ldots$ & +82 & +89 & 53851 & 54104 & $35.281 \pm 0.027$ & $(5 \pm 4) \times 10^{-8}$ & $1.96 \pm 0.005$ \\
\hline $7 \ldots$ & +92 & +107 & 54203 & 54728 & $34.653 \pm 0.009$ & $(6 \pm 5) \times 10^{-7}$ & $1.50 \pm 0.005$ \\
\hline $8 \ldots$ & +109 & +117 & 54758 & 55063 & $34.990 \pm 0.034$ & $(4 \pm 3) \times 10^{-6}$ & $1.98 \pm 0.006$ \\
\hline
\end{tabular}

Note: Cycle number is relative to MJD 50,986.7

${ }^{a}$ CGRO BATSE; ${ }^{b}$ RXTE ASM

to 36.7 days. From the values of $P_{35}$ and $F_{\text {int }}$ shown in Table 1,and Figure 4 it is clear that $P_{35} \propto F_{\text {int }}$ which is in agreement with Leahy and Igna (2010)and the theoretical prediction of Wijers and Pringle (1999). From Figure 3 it is observed that the maximum flux during the short-on state is $\sim 15-20 \%$ of the maximum main-on flux.

The possible mechanism responsible for the ALS and at the same time the change in precession period after each ALS is still not clear and needs to be investigated further. However it is probable that the ALS and change in the precession period after each ALS could be interpreted as a change in the vertical disc structure.

\section{Conclusions}

Since the discovery of the 35-day cycle of Her X-1, it has entered the anomalous low state on seven occasions. The precession periods of Her X-1 after the $2003-2004$ anomalous low state, i.e. the fifth, sixth, seventh and eighth epochs have been determined. It was found that in each case Her X-1 turned on with a new precession period and main-on flux (see Table 1). We further noted that there is a positive correlation between the precession period and the main-on flux, which is a confirmation of the theoretical predictions of Wijers and Pringle (1999).

\section{Acknowledgment}

ASM results were provided ASM/RXTE teams at MIT and RXTE Science Operations Facility and Guest Observer Facility at NASA's Goddard Space Flight Centre.

\section{References}

Giacconi, R., Gursky, H., Kellogg, E., et al. 1973 ApJ, 184, 227

Leahy, D. A. \& Igna, 2010 ApJ 10, 10

Leahy, D. A. \& Marshall, H., 1999 ApJ, 521, 328

Leahy, D. A. \& Scott, D. M., 1998, ApJ 503, L63

Middleditch, J. \& Nelson, J., 1976 ApJ, 208, 567

Reynolds, A. P., Quaintrell, H., Still, M. D., et al. 1997 MNRAS, 288, 43

Scott, D. M., Leahy, D.A.,\& Wilson, R. B., 2000 ApJ, 539, 392

Still, M. \& Boyd, P., 2004 ApJ, 606, L135

Tananbaum, H., Gursky, H., Kellogg, E. M., et al. 1972 ApJ, 174, L145

Wijers, R. A. M. \& Pringle, J. E., 1999 MNRAS, 308, 207 